\title{
A charge-coupled device (CCD) spectrophotometer for measuring marine bioluminescence
}

\author{
A. C. Arneson ${ }^{1}$, S. Benyamin ${ }^{2}$, B. Jones ${ }^{3}$, G. W. Schmidt ${ }^{3}$ \\ ${ }^{1}$ Scripps Institution of Oceanography, University of California San Diego, La Jolla, California 92093, USA \\ 2 Digivision Inc., 5626 Oberlin Dr., San Diego, California 92121, USA \\ ${ }^{3}$ Center for Astrophysics and Space Sciences, University of California San Diego, La Jolla, California 92093, USA
}

\begin{abstract}
We have designed and built a spectrophotometer which detects low intensities of light by means of a charge-coupled device (CCD). The instrument is easy to use and calibrate, and it allows simultaneous spectral ( $3.0 \mathrm{~nm}$ bandwidth) and temporal (10 ms integration time) measurements of flashes from luminescent organisms. The entire operation of the spectrophotometer as well as data acquisition and processing are carried out by an IBM PC AT personal computer. The system is portable, can be used at sea, and could be modified for remote or in situ monitoring of bioluminescent populations. Data from the flashes of bioluminescent dinoflagellate Pyrocystis fusiformis are presented as examples of the resolution and sensitivity of the instrument.
\end{abstract}

\section{INTRODUCTION}

Bioluminescence is common in the surface waters of the ocean (Herring 1977, Lapota \& Losee 1984, Nealson et al. 1985), and even more so in mid-water, where about $75 \%$ of species are capable of light emission (Herring \& Morin 1978). The fact that the wavelengths of luminescent flashes (420 to $560 \mathrm{~nm}$ ) are those optimally transmitted through seawater, and that luminescent organisms have evolved photoreceptors which are maximally sensitive to these wavelengths, suggest that bioluminescence is important in the ecology and behavior of these organisms (Herring 1983).

The chemistry and physiology of bioluminescence, which can be studied in the laboratory, are well understood (e.g. Herring 1978), but our understanding of ecological aspects of bioluminescence is dependent on field studies. To date, field studies have been hindered by the technical problem of detecting and measuring relatively low levels of light in situ as well as by difficulties and costs associated with repeated sampling at sea. To reduce time spent at sea and decrease ambiguities in determining species distributions from net hauls, we have been developing techniques for remotely sensing bioluminescence and for using flash characteristics as a means of species recognition (Nealson et al. 1986).
Knowledge of spectral and temporal characteristics of flashes is a potentially powerful tool in the study of bioluminescence. Although most bioluminescence is centered around the blue-green $(490 \mathrm{~nm}$ ) portion of the visible light spectrum, there is some variation between species (Widder et al. 1983). Technology previously available for the study of spectral characters of bioluminescence required that the light from several flashes be integrated before the signal achieved a level which could be measured by the detector (Wampler \& De Sa 1971, Reynolds 1978). Such studies defined the peak spectral output of the light and the average spectral emission of an organism over several flashes but could not measure color changes within a flash or between dim flashes.

The temporal characters of bioluminescence appear to have more variety than the spectral characters. This temporal component can be complicated and can vary within a single species (Case 1984, Nealson et al. 1986). Typical flash durations for different organisms vary between about $100 \mathrm{~ms}$ and several seconds. Intensities of these flashes are low, but they can differ by as much as several orders of magnitude; at the low end, $2 \times 10^{8}$ photons flash ${ }^{-1}$ is typical for a single, bright dinoflagellate cell. Preliminary studies have shown that by measuring the color- and time-varying components of 
individual flashes it is possible to identify species based on their light emission, i.e. 'luminous fingerprint' (Nealson \& Arneson 1985, Nealson et al. 1986). The ability to identify luminous fingerprints in situ will greatly simplify problems now associated with obtaining information on the ecology and distribution of luminescent organisms. Additionally the ability to measure fine differences in color and kinetics of single flashes may elucidate behavioral uses of light which have so far been difficult to define.

In this paper we describe a spectrophotometer that uses a charge-coupled device (CCD) for a detector. This instrument allows, for the first time, simultaneous high resolution spectral and temporal measurements of bioluminescent events in real time. Up to 64 different wavelength channels with an average bandwidth of 3.0 nanometers ( $\mathrm{nm}$ ) can be measured at integration times from $10 \mathrm{~ms}$ to several seconds. Spectral resolution and integration time are adjustable by means of a camera controller which adjusts effective pixel size and readout rate. The camera controller has 14-bit analog-todigital conversion circuitry and is interfaced with an IBM PC AT personal computer. All data from the CCD spectrophotometer are acquired, stored, and displayed by the computer. We present data from a bioluminescent dinoflagellate to demonstrate the capabilities of the spectrophotometer-computer system.

\section{MATERIAL AND METHODS}

Spectrometer and optics. The spectrometer (Fig. 1) was designed to transmit as much light as possible from a luminescing organism to the CCD detector. Our spectrometer is limited in light through-put by the width of the CCD $(9.6 \mathrm{~mm})$, the desired spectral range $(140 \mathrm{~nm}$ in this case), and the use of conventional optics.

Organisms are confined in a plexiglass aquarium directly adjacent to a $2 \times 6 \mathrm{~mm}$ optical slit. The slit length of $6 \mathrm{~mm}$ can be increased to accommodate slightly larger organisms. Alternatively, a fiber optic (superguide G-UV, Fiberguide Inc.) that terminates in a $2 \times 6 \mathrm{~mm}$ rectangle can be used at the slit to facilitate collection of light from large organisms or isolated photophores. Light passes through the slit to a $200 \mathrm{~mm}$ focal length collimator, which is $31 \mathrm{~mm}$ in diameter. This combination of slit and collimator optimize light input from a flashing organism. The light then passes to a diffraction grating which disperses the spectrum. The grating was selected for peak optical efficiency (about $75 \%$ ) in the blue-green region of the visible spectrum. It is blazed at $17^{\circ} 27^{\prime}$ with 1200 grooves $\mathrm{mm}^{-1}$, and gives optimal performance at about $480 \mathrm{~nm}$, the approximate middle of the range of light emitted by most organisms. The grating can be replaced by a mirror to allow the full undispersed beam to be recorded for organisms producing very little light or to study the detailed temporal evolution of flashes. A camera lens then focuses the image of the slit on to the CCD detector. The camera lens/diffraction grating combination allows dispersed light in the region of 410 to $550 \mathrm{~nm}$ to fill the entire width of the CCD. The camera lens has a focal length of $20 \mathrm{~mm}$ and operates slightly faster than $\mathrm{f} / 1$. The lens is tilted $1.6^{\circ}$ with respect to the $\mathrm{CCD}$ to reduce effects of chromatic dispersion. Image quality at the $\mathrm{CCD}$ is better than the spectral resolution required. The camera and detector are mounted on a common baseplate to allow rotation around the center of the grating to adjust the bandpass received by the $\mathrm{CCD}$. Both the collimator and the

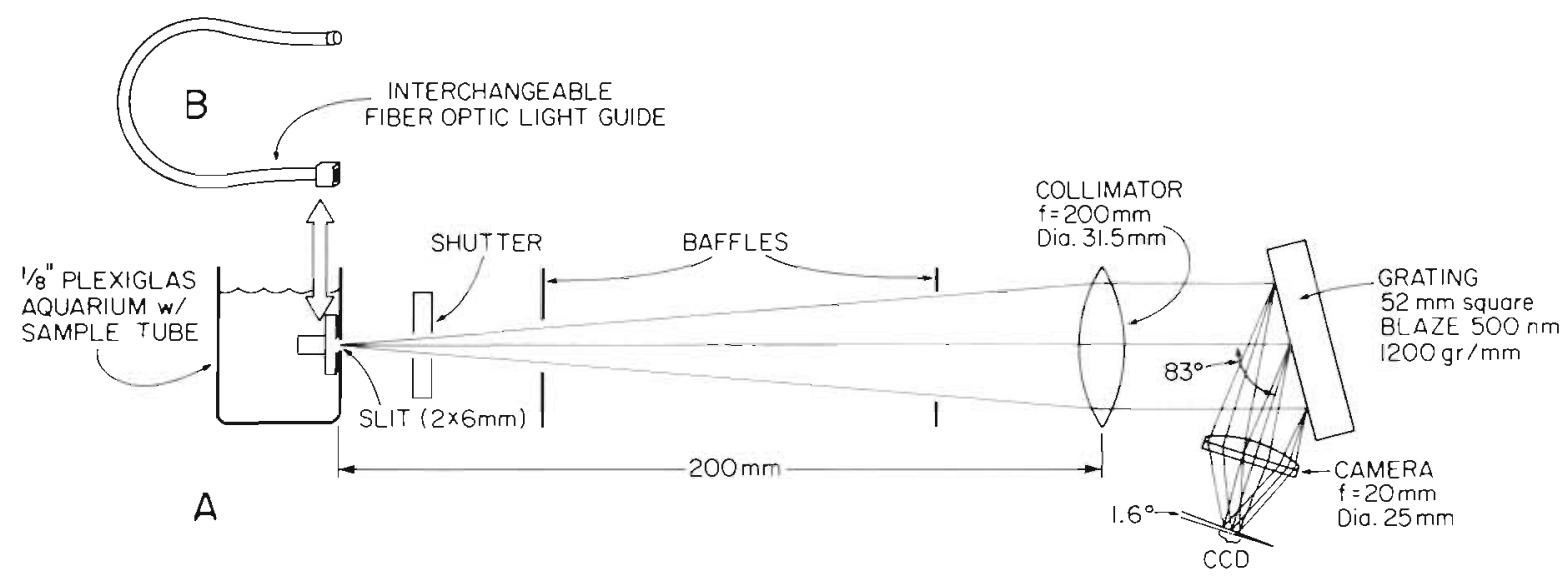

Fig. 1. Block diagram of dispersion optics and sample chamber for the CCD spectrophotometer. (A) Light emitted from a flashing organism at the slit passes through the collimator and then to the diffraction grating where it is dispersed. The dispersed light spectrum from the grating is focused on the CCD by the camera lens. (B) A fiber optic light guide is used as an alternate means of inputting light to the spectrometer. Drawing is to scale 
camera lens have anti-reflection coatings for the $500 \mathrm{~nm}$ region to improve efficiency.

CCD camera. There are several advantages to using a CCD as the detector in our spectrophotometer. First, although the present instrument is designed for laboratory use, our aim is to develop a moored or drifting instrument capable of making in situ measurements. The low power requirements and solid state design of the CCD make it well suited for remote deployment. Second, calibration and multichannel sampling are facilitated by the inclusion of all detector elements on one chip, i.e. essentially one device. Third, the sensitivity is higher and noise is lower than that of conventional photodiodes.

The detector unit (Fig. 2) is based on a Photometrics 81-S fully programable slow scan CCD camera (Photometrics Inc, Tucson, AZ, USA). We have modified this device for low light spectroscopy by moving the CCD closer to the front of the camera housing, using a thinner window in front of the CCD, and replacing the camera optics with our own lens and focus mount. The detector is an RCA SID 501, buried-channel, backsideilluminated CCD. The format of the CCD is $320 \times 512$ pixels, each $30 \times 30 \mu \mathrm{m}$ in size. However, we use only a portion of the CCD by binning pixels and defining the frame size. In effect, part of the $320 \times 512$ pixel format of the CCD is made to look like a linear CCD with $32 \times 2$ large 'superpixels', each consisting of $10 \times 16$ real CCD pixels. By binning pixels we are able to match the detector area to the vertical light output from the spectrometer (512 axis) and vary the spectral resolution (320 axis).

A trade-off exists between spectral resolution and signal-to-noise ratio because the major source of noise is readout noise, which is injected once per superpixel. For most organisms, 32 channels of resolution gives adequate signal above noise. We can increase resolution to 64 elements or higher for very bright flashes. The flexibility to change the active area of the CCD allowed us to try different optical designs in an effort to maximize light collected from flashing organisms. The CCD is optically masked so that only the specified field area is exposed to light. A 4 -stage thermoelectric cooler maintains the $\mathrm{CCD}$ at about $60^{\circ} \mathrm{C}$ below ambient during normal operation. Cooling reduces thermally generated dark noise and optimal working temperatures are achieved by operating the spectrophotometer in an air-conditioned room (about $15^{\circ} \mathrm{C}$ ). The combined effect of masking and cooling assures that an unexposed charge image is read into the optically sensitive region each time an exposed charge image is read out. In this manner the device operates analogously to an unshuttered, sprocketed film slit camera. The time required to advance a frame of data out of the CCD is primarily limited by the signal processing and analogto-digital conversion times of the camera controller and is less than $1 \mathrm{~ms}$ for a 32 element frame.

The camera and spectrometer are mounted together on an anodized aluminum plate, and the whole system except for the organism sample area is housed in a

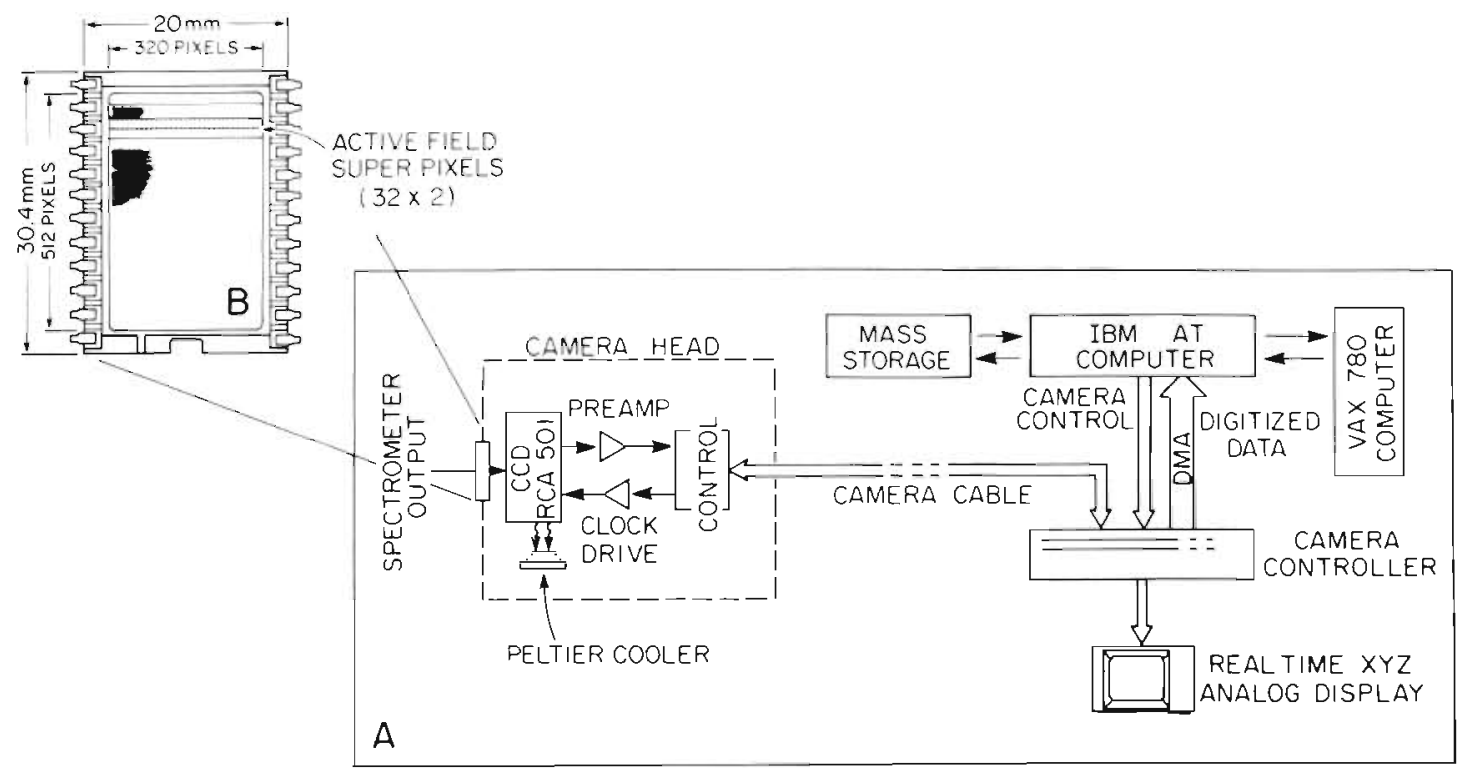

Fig. 2. Block diagram of the $C C D$ camera and the data acquisition processing system. (A) Data signals from the $C C D$ are transferred through the camera controller to the AT computer. Data received by the AT computer can be displayed, stored, and analyzed or sent to a VAX 780 for further data reduction. In the other direction the AT computer sends commands to the camera controller which regulate the CCD camera. (B) Enlarged view of the CCD surface showing the active detector area relative to the size and format of the entire device. Drawing is not to scale 
protective cover. A $5 \mathrm{~m}$ cable connects the camera to the camera controller and allows the instrument to be operated in a separate, darkened room.

Camera control and data acquisition. Timing operations of the camera and part of the camera to computer interface are accomplished with a Model $180 \mathrm{~A}$ digital camera controller (Photometrics Inc.). The image area, binning, sample integration time and read out rate for the CCD are set by inputting commands to the controller. An IBM personal computer AT (or XT) is used to program the controller and acquire data. The computer is connected to the controller through our own interface board. This board is mounted in one of the expansion slots of the personal computer. It uses direct memory access (DMA) to transmit data to the computer, a counter-timer to time acquisition intervals, 2 output ports to program the controller and 2 input ports, one to receive data and the other to sense the status of the controller. A software application program (Fig. 3), written in the system language Forth (PC Forth, Laboratory Microsystems Inc.) controls the entire spectrophotometer system. The program is divided into several sections: camera setup, calibration, data acquisition, 3-dimensional graphic display, file organization, and data reduction.

In the camera setup mode, the program allows the user to define an area of the CCD as the image area and to adjust the binning and sample rates. As part of the setup for data acquisition the user may also specify a trigger threshold level. The trigger looks for 1 to 5 successive values from any channel which are greater than the specified level. In this manner data will only be stored when a flash occurs (i.e. when the output level of the $A / D$ converter is above the desired trigger level). After the camera has been set up it is ready to acquire data. Our standard setup for looking at bioluminescent flashes is 32 channels and an integration time of $30 \mathrm{~ms}$; the trigger level varies with ambient room temperature.

In acquisition mode, data output of the CCD is transferred via DMA to a 64 kilobyte ring buffer. The ring buffer allows infinite acquisition until a trigger event is detected. The size of the data set cannot be bigger than the size of the ring buffer. When an organism flashes in front of the slit of the spectrophotometer the incoming data values become greater than the trigger threshold and a trigger events is registered. After the trigger occurs the program stores a set number of pre-trigger frames and a user-specified number of post-trigger frames. Thus the ring buffer contains the data before, during and after the flash. The program then automatically writes the data to hard disk or waits for user input to save the data set. In overnight data acquisition mode, a file name is automatically assigned to the data set and the system re-initializes to collect another data set. The data sets are stored along with header information as standard DOS (Disk Operating System, Microsoft Inc.) files in a file management directory.

Data sets can be manipulated in several ways, either at the time of acquisition or after the data set has been stored as a file. A 3-dimensional plotting routine allows the user to correct the data using a calibration routine and to view a graphic representation of the data. The plots show time slices before, during, and after the flash; data are displayed as wavelength, time, and intensity. Finally, a data reduction routine can process individual files or groups of files into a reduced data set. For our studies of the flashes of bioluminescent

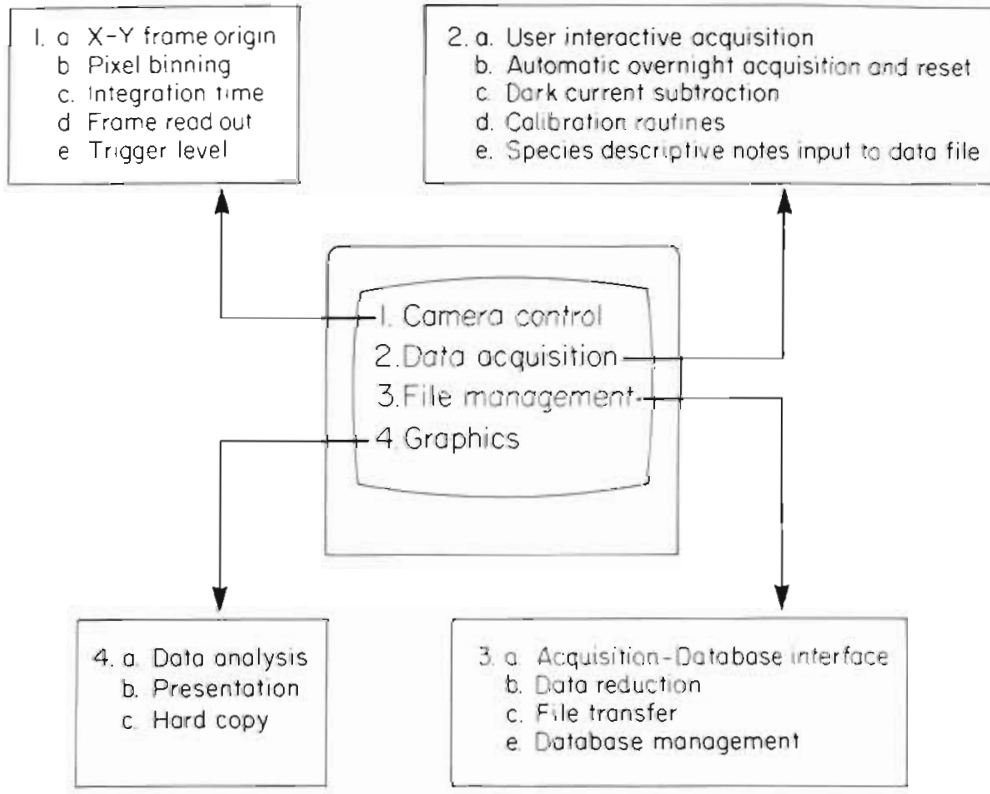

Fig. 3. Block diagram of the spectrophotometer application program. The application program runs on the AT computer and controls all aspects of camera control, data acquisition, file management and graphics 
organisms, the reduction program calculates such parameters as rise time, total time, number of peaks, color distribution, rise and decay rates, and second, third, and fourth moments of the temporal flash curve. Finally, a data management system (R Base system V, Microrim Inc.) organizes the data sets by species and within a species by size, sex, date collected, stimulus, and file number. By linking the database to our data acquisition software a subject's measurements and comments are automatically stored with the raw data from a flash.

\section{RESULTS AND DISCUSSION}

\section{Dynamic range and signal-to-noise ratio}

The CCD is essentially a self-scanned image sensor. Since all charge elements on the device use the same amplifier, variation between detector elements is reduced. The $\mathrm{CCD}$ integrates charge during exposure to light and is then 'read out' on command. The read out process produces an independent signal from each light-sensitive element which is proportional to the light striking that element. Noise enters the system in several ways: the 2 most important are fluctuations in the thermally induced dark current and variations in the output driver stage. The latter is reduced by a dual slope integration technique before the output signal reaches the $\mathrm{A} / \mathrm{D}$ converter and is therefore negligible in our application. To remove pixel to pixel variations in the dark current and to reduce the effect of noise in the background, we read 25 scans from the device after the shutter is closed at the end of each data acquisition session. These scans are averaged for each channel and the resulting values stored with the data for subtraction before data analysis. Occasionally noise enters the system from outside radiation (e.g. cosmic rays), however these noise spikes are large, are of very short duration, and are easily filtered out during data reduction.

The dynamic range of the CCD is set by the fullsignal handling capability of the output stage, the binning parameters and the read out noise. The output stage can handle about 4 full-well pixels, i.e. $\sim 2 \times 10^{6}$ electrons. This means for binning parameters of 10 horizontal by 16 vertical pixels, each superpixel in the linear $C C D$ has an average effective full-well capacity of approximately $1.25 \times 10^{4}$ electrons pixel ${ }^{-1}$. The readout noise for a virtual pixel is approximately 50 electrons, therefore the dynamic range after binning (32 superpixelsj is $\sim 40000: 1$

At room temperatures sensitivity of the CCD is limited by direct fluctuations in thermal dark current associated with temperature. Thermal dark current is reduced by a factor of 2 for about each $8^{\circ} \mathrm{C}$. By cooling the $\mathrm{CCD}$ to below $-50^{\circ} \mathrm{C}$, as in our application, thermal dark current is minimal.

\section{Calibration}

Calibration of the spectrophotometer is accomplished with a 'white balancing' routine to allow spectral components of the data to be corrected for nonuniform wavelength sensitivity of the CCD and absorbtion anomalies of the spectrometer to different wavelengths of light. To calibrate the CCD, the desired read out configuration is specified (usually 32 superpixels). Then the CCD is put in data acquisition mode and illuminated using a spectroradiometric reference light source (Photo Research PR 2303) placed behind the sample aquarium or fiber optic. A correction factor is found such that light distribution measured by the instrument at each channel matches the known spectral output of the white light source.

To assign wavelength values to channel numbers, combinations of narrow band-pass interference filters (Ditric Optics), calibrated with a spectrophotometer (Acta M VI, Bechman Inc.) were placed in front of the white light source. The resulting spectral lines for 24 different wavelengths were mapped to the channel output of the CCD. A didymium-glass filter (SRM 2010, National Bureau of Standards) placed in front of the light source was used to check the wavelength assignments and bandwidth. The measured bandwidth for 32 channels is about $4.5 \mathrm{~nm}$ and for 64 channels is about $3.0 \mathrm{~nm}$. These calibration factors are used with all data collected by the instrument. The calibration is checked periodically, but appears quite stable, even when the spectrophotometer is used aboard ship.

Further calibration tests were run with a mercury vapor lamp and bioluminescent organisms, and results were compared to those obtained from an optical multichannel detection system (Widder et al. 1983). In all cases, the wavelength calibration of the CCD spectrophotometer fell within that of the optical multichannel analyzer for similar bandwidth ranges.

\section{Preliminary application}

The CCD spectrophotometer is being successfully used to measure temporal and spectral properties of bioluminescent flashes, in particular, inter- and intraspecific flash variation in euphausiid shrimp (Huber \& Arneson unpubl.). To exemplify the kind of data obtained with this system, the plots of flashes from individual dinoflagellate cells Pyrocystis fusiformis and $P$. noctiluca are presented (Fig. 4). The flashes of these 


\section{Pyrocystis fusiformis}

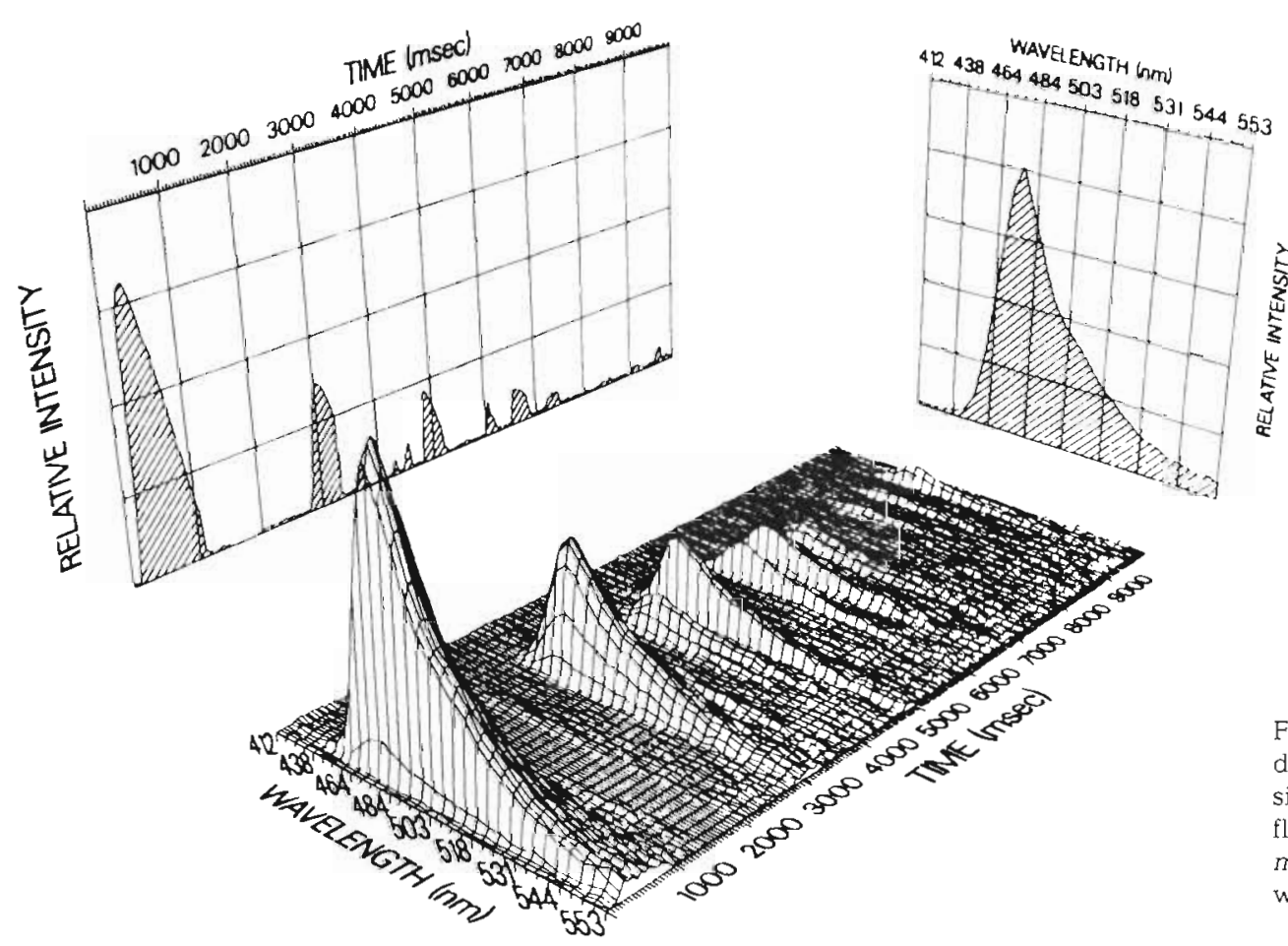

Fig. 4. Temporal and spectral data of the first 5 flashes from a single, dark-adapted dinoflagellate, Pyrocystis fusiformis, are shown as a function of wavelength, time $e^{-1}$, intensity ${ }^{-1}$

organisms have been studied previously (Widder \& Case 1981, Widder et al. 1983), but the data presented here are unique in the sense that both spectral and temporal aspects of single flashes were measured at the same time. Single dinoflagellates produce little light compared to most other organisms and serve as a good test of the sensitivity and resolution attainable using the CCD spectrophotometer.

The ability to analyze spectral and temporal properties at the same time can be a powerful tool in the study of bioluminescence since this information can be used to distinguish different types of bioluminescent organisms (Nealson et al. 1986). We hope to be able to refine variables of flashes so that separate species can be identified from mixed cultures in the laboratory. Ultimately we hope to be able to model the expected behavior of mixed populations so that we can identify species in natural communities. The CCD spectrophotometer with its improved sensitivity, low power requirements and high spectral resolution can easily be adapted for use in situ to study the spatial and temporal distribution of bioluminescent organisms in relation to the physical environment.

Acknowledgements. We thank Dr Ken Nealson of The Center for Great Lake Studies, Milwaukee for support and advice during the initial phases of instrument development. Drs Jim
Case, Eddie Widder and Mike Latz of U.C.S.B. were helpful and generous in letting us cross-calibrate our instrument with theirs and for providing us the opportunity to measure light from the dinoflagellates. We thank Jack Casler, Fred Uhiman, and Arnold Kruse for assistance in design and fabrication of opto-mechanical parts. This work would not have continued without the help of Dr Mark Abbott at Scripps. Dr Mike Huber has helped with many aspects of the project and his involvement is appreciated. This work was supported by a grant from the Office of Naval Research (ONR contract number N 00014$87-\mathrm{K}-0005)$

\section{LITERATURE CITED}

Case, J. F. (1984). Vision in mating behavior of fireflies. In: Lewis, $T$ (ed.) Insect communication. Acadernic Press, New York, p. 195-218

Herring, P. J. (1977). Bioluminescence in marine organisms. Nature, Lond. 267: 788-793

Herring, P. J. (1978). Bioluminescence in action. Academic Press, New York, p. 256

Herring, P. J. (1983). The spectral characteristics of luminous marine organisms. Proc. R. Soc. (Ser. B) 220: 183-217

Herring, P. J., Morin, J. G. (1978). Bioluminescence in fishes. In: Herring, P. J. (ed.) Bioluminescence in action. Academic Press, New York, p. 273-329

Lapota, D., Losee, J. R. (1984). Observations of bioluminescence in marine plankton from the Sea of Cortez. J, exp mar. Biol. Ecol. 77: 209-240

Nealson, K. H., Arneson, A. C. (1985). Marine bioluminescence: about to see the light. Oceanus 29 (3): 13-18 
Nealson, K. H., Arneson, A. C., Bratkovitch, A. (1985). Preliminary results from studies of nocturnal bioluminescence with subsurface moored photometers. Mar Biol. 83: 185-191

Nealson, K. H., Arneson, A. C., Huber, M. E. (1986). Identification of marine organisms using kinetic and spectral properties of their bioluminescence. Mar. Biol. 91. 77-83

Reynolds, G. T (1978). Application of photosensitive devices to bioluminescent studies, Photochem. Photobiol. 27: $405-421$
Wampler, J. E., DeSa, R. (1971). An on-line spectrofluorometer system for rapid collection of absolute luminescence spectra. Appl. Spectrosc. $25(6)$ : 623-627

Widder, E. A., Case, J. F. (1981). Two flash forms in the bioluminescent dinoflagellate, Pyrocystis fusiformis. J. comp. Physiol. 143: 43-52

Widder, E. A., Latz, M. I., Case, J. F. (1983). Marine bioluminescence spectra measured with an optical multichannel detection system. Biol. Bull. mar. biol. Lab., Woods Hole 165: 791-810

This article was presented by Professor N. D. Holland; it was accepted for printing on February 1, 1988 